\title{
Duelo en el cuidador del niño fallecido por cáncer: revisión exploratoria
}

\section{Grief in the caregiver of the child dead by cancer: scoping review}

\author{
Sonia Carreño-Moreno ${ }^{(\mathbb{D})}$, Lorena Chaparro-Díaz ${ }^{2}$, Gloria Mabel Carrillo ${ }^{(\mathbb{D}}$, \\ Olga Janneth Gómez-Ramírez ${ }^{4}$
}

\begin{abstract}
Tipología: Artículo de revisión
Para citar este artículo: Carreño-Moreno S, Chaparro-Díaz L, Carrillo GM, Gómez-Ramírez OJ. Duelo en el cuidador del niño fallecido por cáncer: revisión exploratoria. Duazary. 2019 mayo; 16(2): 281-294. Doi: https://doi. org/10.21676/2389783X.2755
\end{abstract}

Recibido en abril 16 de 2018

Aceptado en diciembre 05 de 2018

Publicado en línea en febrero 15 de 2019

\section{RESUMEN}

El objetivo de esta revisión fue sintetizar el abordaje metodológico de estudio e integrar los hallazgos del proceso de duelo en cuidadores de niños fallecidos por cáncer. Se realizó una revisión exploratoria de literatura de 19 estudios publicados en revistas indexadas entre el 2010 y 2017. Los hallazgos fueron tratados con técnicas de codificación abierta inductiva. Los resultados evidenciaron un abordaje mayormente cualitativo, con participación de padre y madre, con un año entre la muerte del niño y el ingreso al estudio. Los resultados de los estudios revelaron que debido a que el duelo se vive como respuesta a la ruptura física del vínculo con el niño, es a través del trabajo sobre vínculos que el duelo puede superarse. Los vínculos clave son los que se tienen con el niño fallecido, con los hijos vivos, con el cónyuge, con la familia y el equipo de salud; por lo que se requiere acompañamiento continuo y compasivo al cuidador desde el momento del diagnóstico del niño con cáncer, que permita construir vínculos asertivos. Posterior a la muerte del niño, es necesario mantener la continuidad del cuidado y propender por que el cuidador participe en grupos de apoyo.

Palabras clave: duelo; cuidadores; padres; neoplasia; niño; muerte.

\section{ABSTRACT}

The aim of this review was to synthesize the methodological approach that has been studied and integrate the findings of the grief process in caregivers of children who died of cancer. A scoping review was conducted of 19 studies published in journals indexed between 2010 and 2017. The findings were treated with open inductive coding techniques. The results showed a mostly qualitative approach, involving mother and father, with one year between the death of the child and the entry into the study. The results of the studies revealed that because grief is lived as a

1. Universidad Nacional de Colombia. Bogotá, Colombia. Correo: spcarrenom@unal.edu.co - http://orcid.org/0000-0002-4386-6053

2. Universidad Nacional de Colombia. Bogotá, Colombia. Correo: olchaparrod@unal.edu.co - http://orcid.org/0000-0001-8241-8694

3. Universidad Nacional de Colombia. Bogotá, Colombia. Correo: gmcarrillog@unal.edu.co - http://orcid.org/0000-0003-4513-104X

4. Universidad Nacional de Colombia. Bogotá, Colombia. Correo: ojgomezr@unal.edu.co - http://orcid.org/0000-0002-9160-4170 
response to the physical rupture of the bond with the child, it is through the work on bonds that grief can be overcome. The key bonds are those with the deceased child, with the living children, with the spouse, with the family and the health team. Therefore, continuous and compassionate accompaniment of the caregiver is required from the moment of the diagnosis of the child with cancer, which allows the construction of assertive bonds. After the death of the child, it is necessary to maintain the continuity of care and encourage the caregiver to participate in support groups.

Keywords: Grief; Caregivers; Parents; Neoplasms; Child; Death.

\section{INTRODUCCIÓN}

C ada año se diagnostican en el mundo aproximadamente doscientos veinte mil casos nuevos de cáncer infantil, con una supervivencia a cinco años cercana al $80 \%$ en países con ingresos altos, frente a un $10 \%$ en países de ingresos medios y bajos $^{1-3}$.

El diagnóstico de cáncer infantil es un evento inesperado para los cuidadores, para las familias y para la sociedad en general ${ }^{4-6}$. Aunque en general el cáncer infantil tiene un buen margen de curación, la creencia cultural de la asociación entre el cáncer y la muerte hace que los cuidadores de niños con cáncer vivan en un continuo proceso de duelo, pues se enfrentan a diferentes pérdidas durante la trayectoria de la enfermedad, situación que en algunos casos termina con el duelo ante la muerte del niño ${ }^{7-9}$.

No todos los procesos de duelo son iguales, ni entre las diversas situaciones de pérdida, ni entre las personas ${ }^{10-12}$. Los cuidadores familiares de niños fallecidos por cáncer viven un duelo que puede llegar a ser devastador, pues esta se configura en una pérdida de alto valor, por cuanto se trata de una enfermedad que en un niño se percibe como antinatural. Con frecuencia los cuidadores presentan experiencias de duelo complicadas y prolongadas, ya que se enfrentan a la ruptura física y en algunos casos emocional y espiritual de una de las relaciones de apego más estrechas ${ }^{13,14}$.

Ante la muerte de un niño, los cuidadores han informado síntomas depresivos, peor bienestar, problemas de salud y problemas matrimoniales incluso 18 años después de la pérdida ${ }^{13}$, culpa y suicidio $^{15}$, sufrimiento crónico ${ }^{16}$, abuso de alcohol, problemas de crianza y angustia ${ }^{17}$, pérdida de la identidad, aislamiento ${ }^{18}$ y deterioro de las relaciones parentales ${ }^{19,20}$.

El cuidado en oncología pediátrica no termina con la muerte del niño, pues a lo largo de la trayectoria de la enfermedad, los cuidadores del niño establecen una alianza con el equipo de salud, la cual no solo implica el cuidado del niño, sino la configuración en sí mismos como un sujeto de cuidado, situación por la que el proceso de duelo ante la muerte del niño es un aspecto relevante en la práctica y en la investigación ${ }^{21-24}$.

A la luz de la naturaleza de un fenómeno como el duelo en el cuidador familiar del niño fallecido por cáncer, se ha desarrollado investigación que describe características de la vivencia y del proceso, evidencia que permite hacer una revisión con miras a explorar los métodos con los que ha sido estudiada e integrar los hallazgos derivados, lo cual es el objetivo de este estudio. 


\section{MATERIALES Y MÉTODOS}

Revisión exploratoria ${ }^{25}$ de literatura que se desarrolló en las siguientes fases:

Búsqueda sistemática de literatura científica y preselección de estudios primarios. La búsqueda se realizó en las bases de datos LILACS, Sage, Willey online Library, Scopus, Bireme, Scielo, ScienceDirect, PubMed, Cuiden y Proquest con la ecuación de búsqueda (cancer or neoplasms or oncology or tumour or malignancy) AND (children or child or kids) AND ( bereavement or grief or loss ) AND ( caregiver or family or parents $)^{26}$. En este proceso las autoras preseleccionaron los estudios que estaban publicados en revistas indexadas, publicados entre 2010 y 2017, con evidencia de un adecuado manejo ético, con hallazgos reportados acerca del proceso de duelo del cuidador familiar del niño fallecido por cáncer. De este proceso resultaron 63 estudios primarios elegibles.

En cuanto a la lectura crítica y selección, la lectura se realizó por las autoras acorde con los criterios propuestos por Burns y Grove, que incluyen la claridad y concisión del texto, la relevancia social y teórica del problema de investigación, la elección adecuada y aplicación rigurosa del método, el cumplimiento de los principios éticos y el aporte de los hallazgos a la generación, ampliación o contraste de teorías existentes ${ }^{27}$. Los artículos fueron leídos por separado por dos de las autoras; posterior a ello, todas las autoras discutieron los resultados de la lectura de los artículos en cuanto al cumplimiento de criterios de rigor y decidieron en conjunto cuáles ingresarían a la revisión. 19 artículos fueron seleccionados (Figura 1).

Respecto a la extracción, clasificación e integración de hallazgos, acorde con el propósito de la revisión, en el que interesa no solo los hallazgos sino el abordaje metodológico, el proceso se dividió en dos momentos. En el primero, se extrajo la información relevante sobre el abordaje metodológico en una matriz, identificando el diseño del estudio, las características de los participantes, el tiempo mínimo transcurrido entre la muerte del niño y la participación en el estudio y los instrumentos de medición usados. Un ejemplo de dicha matriz se presenta en la tabla 1. En el segundo, se extrajeron los hallazgos relevantes, se les dio un código nominal bajo un sistema de codificación abierta inductiva y mediante la clasificación de códigos y comparación emergieron los temas ${ }^{28}$.

Tabla 1. Descripción de características generales del método de estudio en estudios primarios.

\begin{tabular}{|c|c|c|l|}
\hline Estudio & $\begin{array}{c}\text { Tipo de } \\
\text { estudio }\end{array}$ & Muestra & ¿Se utilizó algún instrumento? ¿Cuál? \\
\hline $\begin{array}{c}\text { Van der Geest } \text { et al } \\
2014\end{array}$ & $\begin{array}{c}\text { Cuantitativo, } \\
\text { descriptivo }\end{array}$ & 87. Padres y madres & Inventory of Traumatic Grief: ITG) \\
\hline $\begin{array}{c}\text { Rosenberg } \text { et al } \\
2014 .\end{array}$ & Mixto & 105. Padres y madres & $\begin{array}{l}\text { Escala de resiliencia Connor - Davidson, } \\
\text { Escala de angustia psicológica Kessler-6, } \\
\text { Inventario de crecimiento postraumático. }\end{array}$ \\
\hline $\begin{array}{c}\text { Nove-Josserand H, } \\
\text { Godet A. 2015. }\end{array}$ & Fenomenología & 15. Padres y madres & Grupo de discusión \\
\hline
\end{tabular}


Tabla 1. Continuación.

\begin{tabular}{|c|c|c|c|}
\hline Estudio & $\begin{array}{l}\text { Tipo de } \\
\text { estudio }\end{array}$ & Muestra & ¿Se utilizó algún instrumento? ¿Cuál? \\
\hline Vega $\mathrm{P}$, et al 2012. & Fenomenología & 8. Padres y madres & Entrevista. \\
\hline $\begin{array}{l}\text { Gerrish NJ, et al } \\
2014 .\end{array}$ & Mixto & 13. Madres & $\begin{array}{l}\text { The Hogan Grief Reaction Checklist-HGRC, } \\
\text { The Post-Traumatic Growth Inventory-PTGI }\end{array}$ \\
\hline $\begin{array}{l}\text { Mullen JE, et al } \\
2015 .\end{array}$ & Estudio de caso & 1. Familia de niña & Entrevista \\
\hline Grinyer, A. 2012. & Cualitativo & 8. Padres y madres & Grupo focal \\
\hline $\begin{array}{l}\text { Welch JG, et al } \\
2012 .\end{array}$ & Mixto & $\begin{array}{l}\text { 31. Padres y madres } \\
\text { de niños }\end{array}$ & $\begin{array}{l}\text { Preguntas sobre datos clínicos del niño } \\
\text { fallecido, Cuestionario de duelo }\end{array}$ \\
\hline $\begin{array}{c}\text { O'Connor K, } \\
\text { Barrera M. } 2014 .\end{array}$ & Cualitativo & 26. Padres y madres & Entrevistas \\
\hline $\begin{array}{l}\text { Darbyshire } \mathrm{P}, \text { et al } \\
2012 .\end{array}$ & Fenomenología & 6. Padres y madres & Entrevistas \\
\hline $\begin{array}{c}\text { Foster TL, et al } \\
2011 .\end{array}$ & Mixto & $\begin{array}{l}36 \text { madres, } 24 \text { padres } \\
\text { y } 39 \text { hermanos }\end{array}$ & Cuestionarios, entrevistas semiestructuradas \\
\hline $\begin{array}{l}\text { Thompson AL, et al } \\
2011 .\end{array}$ & Cualitativo & $\begin{array}{c}65 \text { padres y madres, } y \\
39 \text { hermanos }\end{array}$ & Entrevistas semiestructuradas \\
\hline Alam R, et al 2012. & Cualitativo & 18 madres y 13 padres & Entrevistas semiestructuradas \\
\hline Vega P, et al 2014. & Fenomenología & 3 padres y 5 madres & Entrevistas \\
\hline $\begin{array}{c}\text { Steele AC, et al } \\
2013 .\end{array}$ & Cualitativo & $\begin{array}{c}36 \text { madres, } 24 \text { padres } \\
\text { y } 39 \text { hermanos }\end{array}$ & Entrevistas \\
\hline $\begin{array}{l}\text { Van der Geest et al } \\
2015 .\end{array}$ & Cualitativo & 86 Padres y madres & Cuestionario escrito \\
\hline $\begin{array}{l}\text { Gilmer MJ, et al } \\
2012 .\end{array}$ & Mixto & $\begin{array}{l}36 \text { madres, } 24 \text { padres } \\
\text { y } 39 \text { hermanos }\end{array}$ & Entrevistas semiestructuradas \\
\hline Price J, et al 2011. & Cualitativo & 16 madres y 9 padres & Entrevistas semiestructuradas \\
\hline $\begin{array}{l}\text { Bortz AP, et al } \\
\quad 2013 .\end{array}$ & $\begin{array}{l}\text { Cuantitativo } \\
\text { descriptivo }\end{array}$ & 50 madres & $\begin{array}{l}\text { Cuestionario de información de antecedentes } \\
\text { y cáncer, (TTBQ) (Bereavement } \\
\text { Questionnaire) }\end{array}$ \\
\hline $\begin{array}{l}\text { McCarthy MC, et } \\
\text { al } 2010 .\end{array}$ & $\begin{array}{l}\text { Cuantitativo: } \\
\text { Transversal }\end{array}$ & 58 Padres y madres & $\begin{array}{l}\text { Entrevistas estructuradas, [ICG-R] Inventory } \\
\text { of Complicated Grief-Revised, [BDI-II] Beck } \\
\text { Depression Inventory-Second Edition }\end{array}$ \\
\hline
\end{tabular}

Este estudio cumplió con los criterios éticos normados, considerando que un criterio de selección de los artículos fue el cumplimiento de criterios éticos para la investigación con seres humanos, presentados en la declaración de Helsinki y en las pautas CIOMS. En particular, se verificó que los estudios declararan el proceso de consentimiento informado y la obtención de aval por un comité de ética o investigación. Además, este estudio respetó la propiedad intelectual, haciendo un adecuado proceso de referenciación ${ }^{29}$. 
Figura 1. Proceso de búsqueda y selección de estudios primarios

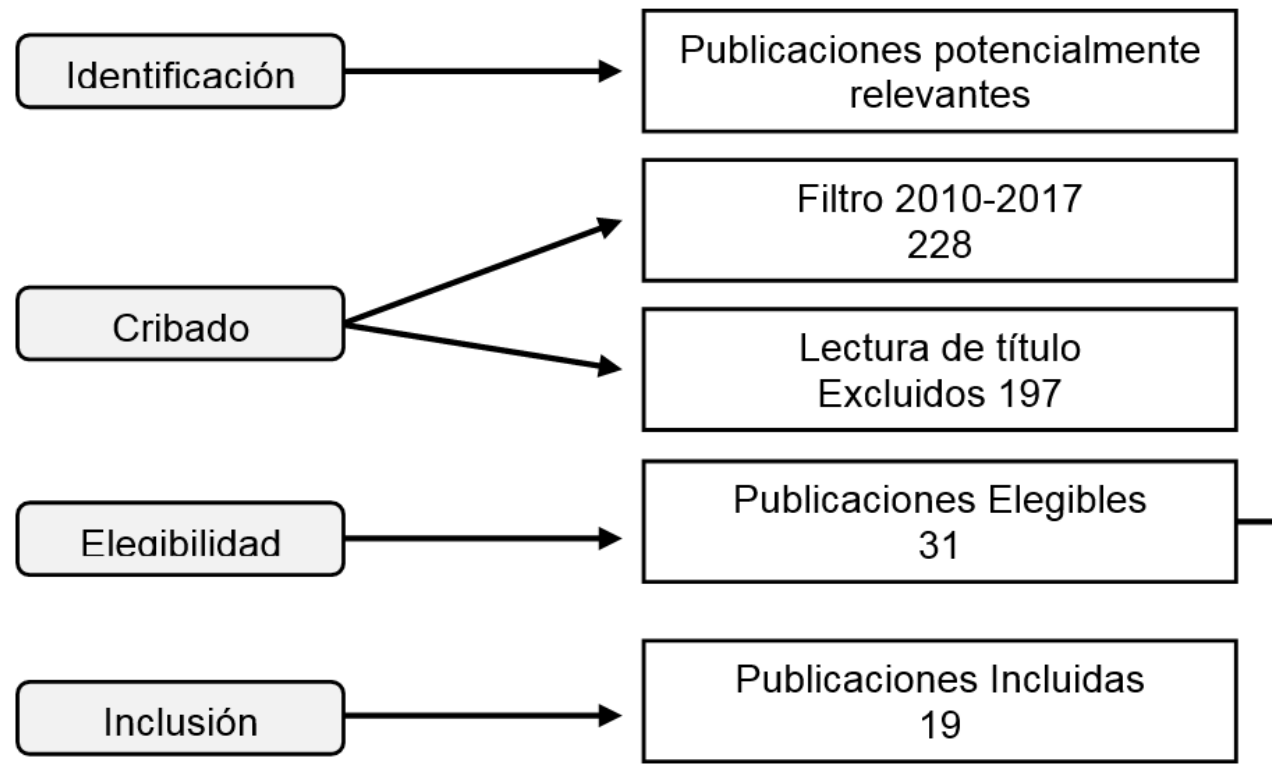

\section{RESULTADOS}

\section{Diseño de los estudios}

El abordaje cualitativo es la tendencia de estudio de este fenómeno, siendo predominante los diseños descriptivos con un $30 \%{ }^{30-35}$, seguidos de los fenomenológicos con un $25 \%^{36-40}$ y un $5 \%$ de estudios de caso $^{41}$. Los diseños mixtos constituyeron el $25 \%{ }^{42-46}$, mientras que los abordajes cuantitativos descriptivos un $15 \%{ }^{47-49}$.

\section{Características de los participantes}

Dentro de los sujetos predilectos para informar acerca del fenómeno se encuentran padre y madre con un $70 \%^{30,32-40,42,44,47,49}$, la familia constituida por padre, madre y hermanos con un $20 \%^{31,41,45,46}$ y por último la madre con un $10 \%{ }^{43,48}$.

\section{Periodo mínimo entre el fallecimiento del niño y el estudio}

El periodo mínimo transcurrido entre el fallecimiento del niño y la inclusión de los informantes a los estudios fue de un año en un $25 \%^{36-38,42,48}$, diez años en un $15 \%^{30,33,47}$, seis meses en un $10 \%{ }^{32,34}$, tres meses en un $5 \%{ }^{46}$, cinco años en un $5 \%{ }^{44}$, veinte meses en un $5 \%^{31}$, un estudio captó a los participantes cuando refirieron creer haber elaborado el duelo ${ }^{39}$ y el $30 \%$ de los estudios no especifica este aspecto ${ }^{35,40,41,43,45,49}$.

\section{Instrumentos de medición}

En los estudios cuantitativos y mixtos se usaron las siguientes medidas para objetivar el fenómeno:

Inventory of Traumatic Grief - ITG ${ }^{47}$, que mide el trastorno de duelo prolongado y trastorno de duelo persistente. Es un instrumento de 29 ítems, con escala Likert de cinco puntos, desde nunca hasta siempre. A mayor puntuación, mayor nivel de duelo.

Kessler- $6^{42}$, que mide la angustia psicológica en general a través de seis ítems, acorde con los criterios DSM-IV. Es una escala ampliamente usada y recomendada por la OMS. 
Post-Traumatic Growth Inventory (PTGI ${ }^{42,43}$, instrumento de 21 ítems que mide los cambios ocurridos luego de eventos negativos. A mayor puntaje, mayor crecimiento. Mide las relaciones con otros, nuevas posibilidades, fuerza personal, cambio espiritual y apreciación de la vida.

The Hogan Grief Reaction Checklist-HGRC ${ }^{43}$, instrumento de 61 ítems diseñado para valorar el duelo positivo y negativo ante la muerte de un ser querido. Mide la desesperación, pánico, ira, culpa, desprendimiento, desorganización y crecimiento personal.

Bereavement Questionnaire (TTBQ) ${ }^{48}$, instrumento de 16 ítems relacionados con el funcionamiento biopsicosocial acorde con la percepción de las madres, que se miden en escala Likert de 5 puntos desde completamente en desacuerdo a completamente de acuerdo. A mayor puntaje mayor funcionamiento.

Inventory of Complicated Grief-Revised ${ }^{49}$, instrumento de 17 ítems que miden el duelo complicado con una escala Likert de 1 a 5 . Mide la ansiedad por separación y el estrés traumático. A mayor puntaje, mayor duelo.

\section{Integración de los resultados}

Los resultados se integraron en cuatro categorías factores que promueven duelo complicado, factores que promueven la resolución del duelo, necesidades de cuidado de los cuidadores y desafíos para estudios futuros. A continuación se desarrolla cada una de las categorías mencionadas.

\section{Factores que promueven duelo complicado}

El proceso de duelo no comienza con la muerte del niño, es un proceso que se desarrolla desde mucho antes, incluso desde el mismo momento del diagnóstico. En este sentido, deficiencias en el cuidado paliativo pueden desencadenar duelo complicado ante la muerte del niño con cáncer; dentro de dichas deficiencias se reporta mala comunicación entre el personal de salud con el niño o la familia, la falta de continuidad en la atención, el abordaje inadecuado de síntomas como fatiga, dolor, ansiedad, soledad y $\mathrm{rabia}^{47}$, mala calidad de vida del niño en sus últimos días $^{49}$, deficiencias en la provisión de información acerca de la gravedad de la enfermedad que no permiten a los cuidadores desarrollar algún tipo de previsibilidad sobre la muerte y aún menos prepararse para este evento ${ }^{43}$ y una percepción negativa de la calidad del cuidado brindado por el equipo de oncología pediátrica ${ }^{49}$.

Las condiciones sociales y económicas de la familia también pueden influir en un duelo complicado. La insatisfacción con el apoyo social recibido, un ambiente social post trauma desprovisto de compañía y consuelo ${ }^{30,43}$ y dificultades económicas en la familia ${ }^{49}$ se han asociado con mayor duelo a largo plazo. Por su parte, el entorno social rutinario de los cuidadores era el hospital, situación que ante la muerte del niño presenta un cambio repentino y rotundo, se presenta una ruptura de las relaciones con el equipo de salud que hace que los cuidadores tengan una sensación de aislamiento, falta de apoyo y abandono ${ }^{38,40}$.

Por último, una capacidad inadecuada para desarrollar respuestas adaptativas por parte de los cuidadores también puede influir en un duelo complicado. Se reportó que situaciones como la afectación en la identidad personal, debido a la pérdida de uno de sus roles ${ }^{36}$, la baja capacidad de estabilizar la identidad ${ }^{30}$, el aislamiento social, la incapacidad de vivir con la idea de que el niño es insustituible ${ }^{36}$, la incapacidad de replantearse positivamente la muerte, crecimiento y propósito personal limitado, la incapacidad para imaginar su propio futuro, la falta de satisfacción en 
la vida, la dificultad para criar hijos vivos y los pensamientos y comportamientos destructivos son factores que pueden asociarse con un duelo complicado $^{30}$.

\section{Factores que promueven la resolución del duelo}

Uno de los aspectos recurrentes reportados por los estudios primarios fue la necesidad de mantener el vínculo con el niño fallecido ${ }^{39,48}$. Durante la trayectoria de la enfermedad, los cuidadores estrechan su relación con el niño, lazo que se puede llegar a estrechar aún más después del fallecimiento, puesto que como ya no existe la necesidad de cuidarlo físicamente, surge la necesidad de estrechar el vínculo interno ${ }^{48}$. Para mantener el vínculo simbólico ${ }^{43}$, los cuidadores realizan prácticas como compartir historias y recuerdos ${ }^{44}$, representaciones visuales, mantener algunas pertenencias personales, pensar en los niños fallecidos, ir a los lugares que ocupaban, organizar actividades en su honor, hacer cosas que a los niños fallecidos les hubiera gustado hacer, visitar cementerios y mantener sus cenizas ${ }^{45}$.

Desarrollar adecuados mecanismos de afrontamiento ante la pérdida favorece la elaboración del duelo. Estudios incluidos en esta revisión informaron que los cuidadores resilientes tenían un mayor crecimiento postraumático ${ }^{42}$, que el proceso de duelo avanza adecuadamente en la medida en que los cuidadores atribuyen sentido a la vida, agonía y muerte de sus hijos, con lo que se facilita la construcción de sentido de la propia vida ${ }^{37,43,48}$, además es clave reconocer los esfuerzos hechos durante la vida del niño, con el fin de evitar ser agobiado por la culpa. ${ }^{32,34}$. Un mediador eficaz de los mecanismos de afrontamiento es la espiritualidad y el mantenimiento de la fe $\mathrm{f}^{31,35-37,39,40}$.

Un ambiente social adecuado es también un aspecto clave a la hora de elaborar el duelo.
Estudios reportan que para hacer frente a las manifestaciones del duelo en padres de niños fallecidos por cáncer, es favorable su inclusión a grupos de apoyo en donde se fomente la interacción con sujetos que hayan pasado por la misma experiencia y se anime a los padres en duelo a seguir viviendo sin olvidar el hecho ocurrido con su hijo ${ }^{35,36}$. En estos grupos, los cuidadores se sienten identificados, consolados, comprendidos y perciben como genuino el apoyo que reciben $^{37}$; con ello, se favorecen momentos de recordación tranquilos y constructivos, en donde es posible la reformulación positiva, la conciencia del crecimiento y propósito personal ${ }^{30}$, la autoexpresión $^{31}$, la reconfiguración de normalidad $^{35}$, el mantenerse ocupado ${ }^{30,40}$, el hablar de la importancia de la aceptación, el continuar con la vida, el vivir cada momento y el centrarse en el presente $^{31}$. Además, buscar la integración social con su familia que aún los necesita y con su red próxima, y ayudar a otras familias en situaciones similares, previene el aislamiento y favorece la resolución del duelo ${ }^{32,40}$.

\section{Necesidades de cuidado de los cuidadores ante el duelo}

Los cuidadores familiares necesitan acompañamiento y apoyo compasivo desde el diagnóstico, la trayectoria de la enfermedad, el cuidado paliativo y por lo menos dos años después de la muerte del niño $30,38,41,44,47$. Los momentos más significativos para el proceso de elaboración de duelo se inician durante la fase paliativa del niño y continúan hasta la despedida, por lo que es importante para los padres sentir que el equipo sanitario hizo todo lo humanamente posible por su hijo ${ }^{37}$. En particular, son necesarias llamadas telefónicas y visitas domiciliarias de parte de los profesionales que hicieron parte del equipo que cuidó al niño durante la enfermedad ${ }^{38,44}$. El apoyo comunitario es clave, orientar a amigos y familiares acerca de cómo brindar apoyo es una herramienta útil, pues a menudo no se sabe 
cómo responder al afligido, el apoyo no solamente se limita a la muerte del niño, sino al restablecimiento de vínculos parentales, matrimoniales y sociales ${ }^{40}$.

Requieren un proceso de comunicación asertiva con los profesionales de salud, centrada en sus necesidades y en la que se permita la participación en la toma de decisiones frente al abordaje del niño ${ }^{41}$. Es imperante hacerlos participes del cuidado paliativo del niño, procurando una comunicación franca frente a pronóstico, los posibles escenarios futuros frente a los síntomas, dependencia y cuidados; además, se debe procurar que tanto hombres como mujeres participen del cuidado directo del niño, siempre con acompañamiento del profesional de salud ${ }^{49}$. Es necesario abrirse a una comunicación honesta sobre cómo se debe hablar con los niños de la muerte $^{33}$, explorar si los padres desean hablar con su niño sobre la muerte ${ }^{30}$ e identificar las razones de los padres para discutir o no discutir sobre la muerte con sus hijos y procurar un entorno propicio para la despedida con el niño fallecido en términos de tiempo y privacidad ${ }^{31,41}$.

Necesitan compañía, consuelo, consejo y mantenerse ocupados, condiciones que pueden darse idealmente en una escenario de interacción con personas significativas como su familia, profesionales de salud que vivieron el proceso de cuidado con ellos y otras personas que, como ellos, saben lo que es perder un hijo a causa del cáncer ${ }^{35-37,39}$. En este escenario, es clave dar soporte físico, psicosocial y espiritual a las familias afligidas ${ }^{41}$, evaluar las dificultades en la expresión del duelo, la relación con los demás y reconocer las diferencias de género en el duelo y el ajuste en hombres y mujeres, considerar los cambios que ocurren en las familias después de la muerte de un hijo, considerando que el cambio no es universal para todas las familias ${ }^{32}$ y estimular la conversación sobre los sentimien- tos y los cambios que experimentan los padres afligidos a fin de brindar herramientas para que estos avancen en su proceso de duelo ${ }^{40,46}$.

\section{Desafíos para estudios futuros}

Los hallazgos de estudios incluidos en esta revisión dieron lugar a una emergente área de estudio dentro de los que se destacan cuatro áreas en diferentes niveles de abordaje metodológico.

Se requiere mayor descripción del concepto de resiliencia como un área estrechamente relacionada con el duelo, futuros estudios pueden comprender la resiliencia bajo técnicas cualitativas, además es un reto diseñar medidas de resiliencia que puedan ser aplicadas en esta población ${ }^{42}$.

En cuanto al duelo propiamente dicho, se requiere mayor descripción acerca de las manifestaciones del proceso de duelo de padres de niños fallecidos por cáncer ${ }^{35}$, ampliar la comprensión de los vínculos continuos entre los padres y los hermanos con los niños fallecidos por cáncer ${ }^{45}$, comprender mejor los patrones de duelo en relación con lo que los cuidadores pueden percibir como ayuda útil a lo largo de su experiencia, describir el papel de la espiritualidad y el significado en los niños y cuidadores en duelo $^{31}$, comprender experiencias en cuidadores con duelo complicado, así como en hermanos y en los mismos profesionales de la salud que proporcionan cuidado ${ }^{39}$, exploración del deseo de los padres de involucrarse en actividades para ayudar a otros, el apoyo de duelo para hermanos, el nivel de contacto con la unidad hospitalaria que puede ser terapéuticamente beneficioso, comportamientos de los padres asociados con el acceso a programas de duelo en el hospital y en la comunidad ${ }^{40}$ y evaluar resultados familiares positivos tras la experiencia de muerte de sus hijos ${ }^{46}$.

En el plano de la comparación, los estudios futuros deben examinar las diferencias de género en el duelo de los padres y el ajuste después de otros 
tipos de muertes infantiles, como accidentes, muerte a causa de otras enfermedades, suicidios y homicidios ${ }^{32}$; comparar en otros miembros de la familia, examinar las similitudes y diferencias entre las culturas en las relaciones con hijos y su asociación con el funcionamiento de padres $^{48}$.

A nivel correlacional, se requiere explorar las relaciones causales entre los síntomas, calidad y continuidad de la atención en cuidados paliativos pediátricos con el bienestar de los cuidadores a largo plazo luego de la muerte del niño ${ }^{47}$, indagar sobre los factores que pueden estar asociados con la tendencia de los padres a mostrar características de reintegración o de desintegración en etapas tanto tempranas como tardías tras la muerte de sus hijos ${ }^{30}$.

Finalmente, se sugiere evaluar la eficacia de intervenciones como visitas domiciliarias o llamadas telefónicas ${ }^{38}$ en el proceso de resolución del duelo ${ }^{44}$.

\section{DISCUSIÓN}

Esta revisión presenta una integración de lo que se conoce frente al duelo del cuidador del niño con cáncer y aspectos relevantes frente a la metodología con la que se ha obtenido dicho conocimiento.

En cuanto a lo metodológico se observa que en concordancia con la naturaleza del fenómeno, el abordaje es principalmente descriptivo ${ }^{30-40}$, con mayor tendencia hacia lo cualitativo; sin embargo, es llamativa la tendencia a desarrollar estudios con diseños mixtos. Además, se observa cómo en este tema en particular uno de los desafíos es trascender de lo descriptivo a lo predictivo y prescriptivo ${ }^{30,32,38,44,47,48}$. Se observa un avance importante en la generación de conocimiento en el área, reflejado en que el duelo cuenta con cuatro instrumentos específicos para su medición ${ }^{43,47-49}$.
En cuanto a los informantes, se reportó que son principalmente los padres y madres ${ }^{30,32-40,42,44,47,49}$, con una tendencia importante a incluir también a hermanos ${ }^{31,41,45,46}$. Sin embargo, uno de los ámbitos de investigación necesarios es la comparación de la vivencia por género, pues se presume que es una variable predictora del proceso ${ }^{32}$.

En el proceso de duelo, el tiempo es una variable influyente, condición que consideraron la mayoría de estudios incluidos en esta revisión. Se observó una tendencia a incluir informantes con al menos un año de tiempo entre el fallecimiento del niño y la participación en el estudio ${ }^{36-38,42,48}$.

En concordancia con la naturaleza del duelo como la ruptura de un vínculo por lo menos en el plano físico, se observó como un hilo conductor en los hallazgos que es a través de la intervención en los vínculos que el duelo puede ser resuelto o puede configurarse en algo complicado y prolongado. En particular, el vínculo con el niño con cáncer, el cual es una construcción previa a la enfermedad, que se estrecha progresivamente a medida que esta avanza y que es necesario promover haciendo participes a los cuidadores del cuidado paliativo y de la toma de decisiones. Es fundamental comprender que la resolución del duelo no está en el olvidar la pérdida o minimizarla, sino en construir un significado de la importancia de la vida del niño y de la vida propia ${ }^{39,43-45,48}$, como un punto de partida para poder reestablecer las relaciones con otros significativos como el conyugue, los demás hijos y la red social próxima ${ }^{40}$. La necesidad de trabajar sobre las relaciones es un hallazgo similar a lo reportado en cuidadores que han perdido un niño por otras enfermedades ${ }^{18,34,50,51}$.

El vínculo con los profesionales también es un aspecto central, con la partida del niño se viven otras pérdidas, entre ellas la familia que se construyó con el equipo de salud del centro oncoló- 
gico, situación por la que es necesario mantener la comunicación y acompañamiento a los cuidadores por lo menos hasta dos años después de la muerte del niño ${ }^{30,38,41,44,47}$. En el proceso de mantenimiento de un vínculo positivo con el niño fallecido y el fortalecimiento o reconstrucción del vínculo con otros significativos es útil el desarrollar grupos de apoyo en los que, a través de la interacción con pares y con profesionales de salud, el cuidador se sienta acompañado y comprendido, lo que puede favorecer la adaptación y aceptación de la pérdida ${ }^{32,35-37,39-41,46}$.

Por otra parte, se observó que el duelo en esta población está ampliamente descrito; sin embargo, es necesario expandir la descripción en temas como la espiritualidad, el significado y los vínculos continuos ${ }^{31,45}$; además, es requerida investigación para determinar las variables predictoras del duelo complicado, comparar los procesos de duelo entre diferentes enfermedades y poblaciones ${ }^{32,48}$ y avanzar en el diseño y puesta a prueba de intervenciones ${ }^{38,44}$.

\section{CONCLUSIÓN}

La investigación en el duelo del cuidador del niño con cáncer requiere trascender de la descripción hacia estudios en los que se examinen las variables que predicen el duelo complicado o prolongado, además del diseño y prueba de intervenciones para prevenirlo o mitigarlo.

El trabajo continuo y comprehensivo sobre los vínculos es un aspecto central en la prevención y abordaje del duelo en el cuidador del niño fallecido por cáncer. Es indispensable desarrollar programas de cuidados paliativos en los que se propenda por la participación de los cuidadores en el cuidado directo y en la toma de decisiones; durante el proceso de muerte es necesario mantener las creencias y adecuar un escenario propicio para la despedida, y posterior a la muerte del niño es imperante continuar el abordaje de los cuidadores en especial a partir de grupos de apoyo fuera del hospital.

\section{DECLARACIÓN SOBRE CONFLICTOS DE INTERESES}

Los autores no declaran ningún conflicto de interés.

Este producto fue realizado con la financiación de la Universidad Nacional de Colombia, SIUN código 34625.

\section{REFERENCIAS BIBLIOGRÁFICAS}

1. Siegel R, Miller K, Jemal A. Cancer statistics, 2018. CA Cancer J Clin [Revista en la internet]. 2018 [citado 2018 Feb 5]; 68(1): 7-30. Doi: http:// doi.wiley.com/10.3322/caac.21442.

2. International Agence for Research on Cancer. International incidence of childhood cancer [Internet]. [citado 2018 Jun 1]. Disponible en: http://iicc.iarc.fr/about/iicc3_series.php

3. International Agence for Research on Cancer. GLOBOCAN 2012 [Internet]. [citado 2018 Jun 18]. Disponible en: http://globocan.iarc.fr/Pages/ fact_sheets_cancer.aspx

4. Patel S, Fernandez N, Wong A, Mullins W, Turk A, Dekel N, et al. Changes in self-reported distress in end-of-life pediatric cancer patients and their parents using the pediatric distress thermometer. Psychooncology [Revista en la Internet]. 2014 [Citado 2018 Jun 1]; 23(5): 592-596. Doi: http:// doi.wiley.com/10.1002/pon.3469. 
5. Nam G, Warner E, Morreall D, Kirchhoff A, Kinney A, Fluchel M. Understanding psychological distress among pediatric cancer caregivers. Support Care Cancer [Revista en la Internet]. 2016 [citado 2018 Jul 16]; 24(7): 3147-3155. Doi: http://doi.org/10.1007/ s00520-016-3136-Z

6. Lau S, Lu X, Balsamo L, Devidas M, Winick N, Hunger S, et al. Family life events in the first year of acute lymphoblastic leukemia therapy: a children's oncology group report. Pediatr Blood Cancer. 2014;61(12): 2277-2284. Doi: http://dor. org/10.1002/pbc.25195.

7. Dokken D. Making Meaning after the Death Of a Child: Bereaved Parents Share Their Experiences. Pediatr Nurs. 2013; 39(3): 147-50.

8. Rosenberg A, Dussel V, Kang T, Geyer J, Gerhardt C, Feudtner C, et al. Psychological Distress in Parents of Children With Advanced Cancer. JAMA Pediatr [Revista en la Internet]. 2013 [citado 2018 Jun 1]; 167(6): 537. Doi: http// doi.org/10.1001/jamapediatrics.2013.628

9. Yoshida S, Amano K, Ohta H, Kusuki S, Morita T, Ogata A, et al. A comprehensive study of the distressing experiences and support needs of parents of children with intractable cancer. Jpn J Clin Oncol [Revista en la Internet]. 2014 [citado 2018 Jun 1]; 44(12):1181-1188.Doi: http://doi. org/10.1093/jjco/hyu140.

10. Friedrichsdorf S, Postier A, Dreyfus J, Osenga K, Sencer S, Wolfe J. Improved Quality of Life at End of Life Related to Home-Based Palliative Care in Children with Cancer. J Palliat Med [Revista en la Internet]. 2015 [citado 2018 Jun 1]; 18(2): 143-150. Doi: http://doi.org/10.1089/ jpm.2014.0285.

11. Lützau P, Hechler M, Metzing S, Wolfe J. Children Dying from Cancer: Parents' perspectives on symptoms, quality of life, characteristics of death, and end-of-life decisions. J Palliat Care. 2012; 28(4): 274-281.

12. Robert R, Zhukovsky D, Mauricio R, Gilmore K, Morrison S, Palos G. Bereaved Parents' Perspectives on Pediatric Palliative Care. J Soc Work End Life Palliat Care [Revista en la Internet]. 2012 [citado 2018 Feb 5]; 8(4): 316-338. Doi: http://doi.org/10. 1080/15524256.2012.732023.

13. Rogers C, Floyd F, Seltzer M, Greenberg J, Hong J. Long-term effects of the death of a child on parents' adjustment in midlife. J Fam Psychol [Revista en la Internet]. 2008 [citado $2018 \mathrm{Feb}$ 5]; 22(2): 203-211. Doi: http://doi.org/10.1080/1 5524256.2012 .732023 .

14. Cacante C, Arias Valencia M. Asking for support: the case of families with children suffering cancer. Investig y Educ en Enfermería. 2009 jul; 27(2): 170-180. Disponible en: http://www. scielo.org.co/scielo.php?script=sci_arttext $\&$ pi $\mathrm{d}=$ S0120-53072009000200002

15. Zetumer S, Young I, Shear M, Skritskaya N, Lebowitz B, Simon N, et al. The impact of losing a child on the clinical presentation of complicated grief. J Affect Disord [Revista en la Internet]. 2015 [citado 2018 Feb 5]; (170): 15-21. Doi: http://doi.org/10.1016/j.jad.2014.08.021.

16. Buckley C. When Sorrow Never Stops. Chronic Sorrow After the Death of a Child. J Christ Nurs [Revista en la internet]. 2016 [citado 2018 Feb 5]; 33(1): 22-25. Disponible en: http://www.ncbi. nlm.nih.gov/pubmed/26817366

17. Sandler I, Tein J, Cham H, Wolchik S, Ayers T. Long-term effects of the Family Bereavement Program on spousally bereaved parents: Grief, mental health problems, alcohol problems, and coping efficacy. Dev Psychopathol [Revista en la Internet]. 2016 [citado 2018 Feb 5]; 28(3): 801-818. Doi: http://dpi.org/10.1017/ S0954579416000328 
18. Price J, Jones A. Living Through the Life-Altering Loss of a Child: A Narrative Review. Issues Compr Pediatr Nurs [Revista en la Internet]. 2015 [citado 2018 Feb 5]; 38(3): 222-240. Doi: http://dpi.org/10.3109/01460862.2015.1045102.

19. Joronen K, Kaunonen M, Aho A. Parental relationship satisfaction after the death of a child. Scand J Caring Sci [Revista en la Internet]. 2016 [citado 2018 Feb 5]; 30(3): 499-506. Doi: http:// doi.org/10.1111/scs.12270.

20. DeCinque N, Monterosso L, Dadd G, Sidhu R, Macpherson R, Aoun S. Bereavement support for families following the death of a child from cancer: experience of bereaved parents. J Psychosoc Oncol. 2006; 24(2): 65-83. Doi: http:// doi.org/10.1300/J077v24n02_05

21. Lichtenthal W, Corner G, Sweeney C, Wiener L, Roberts K, Baser R, et al. Mental Health Services for Parents Who Lost a Child to Cancer: If We Build Them, Will They Come? J Clin Oncol [Revista en la Internet]. 2015 [citado 2018 Jun 2]; 33(20): 2246-2253. Doi: http://dpi.org/10.1200/ JCO.2014.59.0406

22. Williams A, McCorkle R. Cancer family caregivers during the palliative, hospice, and bereavement phases: a review of the descriptive psychosocial literature. Palliat Support Care [Revista en la Internet]. 2011[citado 2018 Jun 20]; 9(3): 315-325. Doi: http://doi.org/10.1017/ S1478951511000265

23. Rosenberg A, Baker K, Syrjala K, Wolfe J. Systematic review of psychosocial morbidities among bereaved parents of children with cancer. Pediatr Blood Cancer [Revista en la Internet]. 2012 [citado 2018 Jun 2]; 58(4): 503-512. Doi: http://doi.org/10.1002/pbc.23386

24. Grassi L. Bereavement in families with relatives dying of cancer. Curr Opin Support Palliat Care [Revista en la Internet]. 2007 [citado 2018 Jun
20];1(1): 43-49. Doi: http://doi.org/10.1097/ SPC.0b013e32813a3276

25. Manchado R, Tamames S, López M, Mohedano L, D'Agostino M, Veiga de Cabo J. Revisiones Sistemáticas Exploratorias- Scoping review. Med Segur Trab (Madr) [Revista en la Internet]. 2009 [citado 2018 Feb 5]; 55(216): 12-19. Disponible en: http://scielo.isciii.es/scielo.php?script $=$ sci_ arttext\&pid=S0465-546X2009000300002

26. Sanz J, Veiga de Cabo J, Rojo C, D'Agostino M, Wanden C, Espulgues Pellicer J, et al. Los filtros metodológicos: aplicación a la búsqueda bibliográfica en la medicina del trabajo española. Med Segur Trab. 54(211): 75-83. Disponible en: http://scielo.isciii.es/scielo.php?script $=$ sci_ arttext\&pid=S0465-546X2008000200009

27. Burns N, Grove S. Investigación en enfermería. Quinta edición. España: editorial Elsevier Ltd; 2012.

28. Cáceres P. Análisis cualitativo de contenido: una alternativa metodológica alcanzable. Psicoperspectivas [Revista en la Internet]. 2003 [citado 2017 Dec 27]; 2: 53-82. Disponible en: http://www.psicoperspectivas.cl/index.php/ psicoperspectivas/article/viewFile/3/3

29. Congreso de Colombia. LEY 44 DE 1993 [Internet]. Congreso de Colombia. 1993 [citado 2015 Jul 14]. Disponible en: http://cdr.com.co/ phocadownload/ley 44 de 1993.pdf

30. O'Connor K, Barrera M. Changes in parental self-identity following the death of a child to cancer. Death Stud [Revista en la Internet]. 2014; 38(6):404-411. Disponible en: http://www.ncbi. nlm.nih.gov/pubmed/24666147. doi.org/10.108 0/07481187.2013.801376

31. Thompson A, Miller K, Barrera M, Davies B, Foster T, Gilmer M, et al. A Qualitative Study of Advice From Bereaved Parents and Siblings. J Soc Work End Life Palliat Care [Revista en la Internet]. 2011; 7(2-3):153-172. Doi: http://doi. org/10.1080/15524256.2011.593153 
32. Alam R, Barrera M, D’Agostino N, Nicholas D, Schneiderman G. Bereavement Experiences of Mothers and Fathers Over Time After the Death of a Child Due to Cancer. Death Stud. 2012;36(1): $1-22$.

33. Van der Geest I, Van Den Heuvel M, Van Vliet L, Pluijm S, Streng I, Michiels E, et al. Talking about Death with Children with Incurable Cancer: Perspectives from Parents. J Pediatr [Revista en la Internet]. 2014; 167(6): 1320-1326. Doi: http://doi.org/10.1016/j.jpeds.2015.08.066

34. Price J, Jordan J, Prior L, Parkes J. Living through the death of a child: A qualitative study of bereaved parents' experiences. Int J Nurs Stud [Revista en la Internet]. 2011;48(11):1384-1392. Doi: http:// dx.doi.org/10.1016/j.ijnurstu.2011.05.006.

35. Grinyer A. A bereavement group for parents whose son or daughter died from cancer: how shared experience can lessen isolation. Mortal Promot Interdiscip study death dying. 2012;17(4): 338-354. Doi:http://doi.org/10.1080 /13576275.2012.730684

36. Nove-Josserand H, Godet A. La mort d'un enfant: un réel absolu, irréversible et sans appel ?: expériences groupales découte et de suivi de parents ayant perdu un enfant d'un cance. Psycho-Oncologie. 2015; 9(3): 190-196. Doi: http://doi.org/10.1007/s11839-015-0531-5

37. Vega P, Rivera S, González R. El transitar del sobrevivir al revivir: Comprender la vivencia de padres y madres que han perdido a sus hijos a causa del cáncer. Rev Chil Pediatr. 2012; 83(3): 247-257. Doi:http://doi.org/10.4067/ S0370-41062012000300006

38. Darbyshire P, Cleghorn A, Downes M, Elford J, Gannoni A, Mccullagh C, et al. Supporting bereaved parents: A phenomenological study of a telephone intervention programme in a paediatric oncology unit. J Clin Nurs.
2013; 22(3-4): 540-549. Doi: http://doi. org/10.1111/j.1365-2702.2012.04266.x.

39. Vega P, Rivera M, Gonzalez R. When grief turns into love: understanding the experience of parents who have revived after losing a child due to cancer. J Pediatr Oncol Nurs [Revista en la Internet]. 2014; 31(3): 166-176. Doi: http://doi. org/10.1177/1043454213515547

40. Snaman J, Kaye E, Torres C, Gibson D, Baker J. Parental Grief Following the Death of a Child from Cancer: The Ongoing Odyssey. Pediatr Blood Cancer [Revista en la Internet]. 2016 [citado 2018 Feb 5]; 63(9):1594-1602. Doi: http://doi.org/10.1002/pbc.26046.

41. Mullen J, Reynolds M, Larson J. Caring for pediatric patients' families at the child's end of life. Crit Care Nurse. 2015; 35(6):46-56. doi: 10.4037/ccn201561442. Rosenberg A, Starks H, Jones B. "I know it when I see it." The complexities of measuring resilience among parents of children with cancer. Support Care Cancer. 2014; 2661-2668. Doi: http://doi. org/10.1007/s00520-014-2249-5

42. Gerrish N, Neimeyer R, Bailey S. Exploring maternal grief: A mixed-methods investigation of mothers' responses to the death of a child from cancer. J Constr Psychol [Revista en la Internet]. 2014; 27(3): 151-173. Doi: http://doi.org/10.108 $0 / 10720537.2014 .904700$

43. Welch J, Mannix M, Boergers J, Jelalian E, Barbosa F, Fujii-Rios H, et al. Parental interest in a bereavement support visit when a child dies from cancer. Omega (United States) [Revista en la Internet]. 2012; 65(4): 335-346. DOi: http:// doi.org/10.2190/OM.65.4.f

44. Foster T, Gilmer M, Davies B, Dietrich M, Barrera $\mathrm{M}$, Fairclough L, et al. Comparison of Continuing Bonds Reported by Parents and Siblings After a Child's Death from Cancer. Death Stud. 2011; 35: 420-440. Doi: http://doi.org/10.1080/07481187. 2011.553308 
45. Gilmer M, Foster T, Vannatta K, Barrera M, Davies B, Dietrich M, et al. Changes in parents after the death of a child from cancer. J Pain Symptom Manage [Revista en la Internet]. 2012; 44(4): 572-582. Doi: http://doi.org/10.1016/j. jpainsymman.2011.10.017

46. Van Der Geest I, Darlington A-SE, Streng I, Michiels E, Pieters R, Van Den Heuvel-Eibrink M. Parents' experiences of pediatric palliative care and the impact on long-term parental grief. J Pain Symptom Manage [Revista en la Internet]. 2014; 47(6): 1043-1053. Doi: http:// doi.org/10.1016/j.jpainsymman.2013.07.007

47. Peles Bortz A, Malkinson R, Krulik T. Bereaved Jewish Mothers of Children Who Died of Cancer. Cancer Nurs [Revista en la Internet]. 2013; 36(5): E1-8. Doi: http://doi.org/10.1097/ NCC.0b013e31827eecab.

48. McCarthy M, Clarke N, Ting CL, Conroy R, Anderson V, Heath J. Prevalence and predictors of parental grief and depression after the death of a child from cancer. J Palliat Med [Revista en la
Internet]. 2010; 13(11): 1321-1326. Doi: http:// doi.org/10.1089/jpm.2010.0037

49. Albuquerque S, Narciso I, Pereira M. Dyadic coping mediates the relationship between parents' grief and dyadic adjustment following the loss of a child. Anxiety, Stress Coping [Revista en la Internet]. 2018 [citado 2018 Feb 5]; 31(1): 93-106. Doi: http://doi.org/10.1080/10615806.2 017.1363390

50. Infurna F, Luthar S. Parents' adjustment following the death of their child: Resilience is multidimensional and differs across outcomes examined. J Res Pers [Revista en la Internet]. 2017 [citado 2018 Feb 5]; (68): 38-53. Doi: https://doi.org/10.1016/j.jrp.2017.04.004

51. Infurna F, Luthar S. Parents' adjustment following the death of their child: Resilience is multidimensional and differs across outcomes examined. J Res Pers [Revista en la Internet]. 2017 [citado 2018 Feb 5]; (68): 38-53. Doi: https://doi.org/10.1016/j.jrp.2017.04.004 